\title{
Ueber Anus suburethralis, seine Entstehung und Belandlung.
}

\author{
Von \\ Dr. Robert Ziegenspeck, \\ Docent für Gynäkologie in München.
}

(Mit 3 Abbildungen.)

Am 8. April 1886 gegen Morgen wurde in der UniversitätsFrauenklinik zu Jena ${ }^{1}$ ) ein Knabe geboren, welcher am folgenden Tage Meconium entleerte, und zwar aus einer Oeffnung, die sich einen Centimeter von der Wurzel des Penis entfornt auf dessen Unterseite befand.

Verfasser war bei der Geburt selbst zugegen. Sie erfolgte aus erster Schädellage, das Kind schrie sogleich mit kräftiger Stimme und trug alle Zeichen der Reife. Es hatte $51 \mathrm{~cm}$ Länge und $3585 \mathrm{~g}$ Gewicht. Zwei Verbildungen desselben fielen sofort auf. Zuerst fand sich die Endphalanx des rechten Daumens doppelt vorhanden, dann zeigte sich die Raphe perinei stark ausgeprägt, die Raphe scroti dagegen tief eingesunken; beide zeigten buckelförmige, vorgebuchtete, einer varicösen Vene an Form und Farbe sehr ähnliche Stellen. Eine genauere Untersuchung wurde erst bei Tageslicht vorgenommen.

Am Vormittage des nachfolgenden Tages fand sich ein wenig Meconium an der oben bezeichneten Stelle an der Unterseite des Penis, ungefähr $1 \mathrm{~cm}$ von dessen Wurzel entfernt. Die daselbst befindliche Oeffnung war nur für den Knopf einer $1 / 2 \mathrm{~mm}$-Sonde zugänglich. Die Sonde gelangte in einen Kanal, welcher zunächst dicht unter der Haut verlief, dann aber in die erste jener varixähnlichen Stellen, welche sich etwa $2 \mathrm{~cm}$ aufwärts vor der kleinen

1) Geburtsjournale 1886, Nr. 3. 
. Mündung an der Raphe scroti befand. Hier war die Bedeckung so dünnwandig, dass man den Sondenknopf durch die Haut hindurchschimmern sah. Von da ab verlief der Kanal tiefer, im Maximum $1 \frac{1 / 2}{\mathrm{~cm}}$ tief von der Oberfläche entfernt, im Septum scroti, um am hinteren Ende des Scrotum wiederum in einen derartigen varixähnlichen Hohlraum zu gelangen. Von da ab verlief er oberflächlich in der Raphe scroti, von einer undurchsichtigen Epidermis bedeckt; nur an jener Stelle, wo sich sonst der After befindet, befand sich ein dritter, und zwar grösserer Hohlraum, welcher jedoch mit einem weniger durchsichtigen Integument bedeckt war. Hier fühlte man, dass der Kanal knieförmig aufwärts abgebogen war und offenbar in _das Rectum einmündete.

Dies ist die Missbildung, um welche es sich handelt. Ausserdem fand sich noch das Praeputium mit der Glans penis verwachsen, so dass gar keine Corona glandis gebildet war. Im Uebrigen war das Kind wohlgebildet und gesund.

Die Mutter war eine kräftige, wohlgebildete Drittgebärende in mittleren Jahren, die Frau eines Arbeiters, welche wegen mangelnder häuslicher Pflege die Anstalt aufgesucht hatte. Sie war stets gesund gewesen, auch bestand keine Blutsverwandtschaft zwischen den Ehegatten. Auch der Ehemann war gesund und zeigte keinerlei Entwickelungsfehler. Indessen muss hervorgehoben werden, dass schon das zweitgeborene Kind mit überzähligen Daumen zur Welt gekommen war. Das erste Kind war wohlgebildet.

Auch in der dritten Schwangerschaft hatte die Mutter sich vollkommen wohl befunden und war auch durch die Untersuchung weder in den Respirations-, noch Circulations-, noch Verdauungs-, noch Harn- und Geschlechtsorganen eine auffallende gröbere Veränderung gefunden worden. Höchstens dürfte erwähnt worden, dass die drei Zeichen des Morbus Basedowii in mässigem Grade, aber deutlich vorhanden waren, so dass ich schon vor der Geburt, also bevor ich bestrebt war, für den Bildungsfehler des Kindes eine Ursache zu finden, einen Collegen darauf aufmerksam machte. Ich führe diesen Umstand der Vollständigkeit wegen an, ohne viel Werth darauf zu legen, da ja Vergrösserung der Schilddrüse regelmässig in der Schwangerschaft sich findet und auch Exophthalmus mit Herzklopfen sich oft genug finden liesse, wenn man darauf achten würde. Indessen hatte die Frau, wie sie nachträglich angab, während der ganzen Schwangerschaft Angst davor empfunden, dass auch das dritte Kind, wie das zweite, mit doppelten 
Daumen geboren werden möchte. Das Kind wurde gestillt und. das Wochenbett verlief ohne Störung.

Es handelte sich nun um die Frage: was soll mit der Anusmissbildung geschehen? Die Chirurgen pflegen nur dann Operationen an Neugeborenen vorzunehmen, wenn eine Gefahr dazu zwingt, andernfalls zu warten, bis die Kinder grösser und widerstandsfähiger geworden sind. Die Haut der Neugeborenen neigt zu Ekzemen und Wundsein, verträgt daher schlecht einen Verband. Sie ist anfangs in reichlicher Abschuppung begriffen. Daher erfordert es die Reinlichkeit, dass das Kind täglich gebadet werde, wobei leicht eine Infection der Wunde entstehen kann. Alles das gilt in besonders hohem Grade-von derjenigen Gegend, wo in unserem Falle die Operation hätte vorgenommen werden müssen. Aus diesem Grunde wurde beschlossen abzuwarten.

Es entleerte sich nun reichlich Meconium aus der Oeffnung. Das Kind nahm die Brust und schlief ohne Zeichen eines Missbehagens. Mehrmals war Urinentleerung aus der natürlichen Mündung der Harnröhre an der Spitze des Penis direct beobachtet worden. Derselbe enthielt viel Harnsäure.

Erst am Abende des folgenden Tages fiel eine unbedeutende Auftreibung des Leibes auf, welche sich bis zum Mittage des dritten Tages, des 10. April, steigerte und allmälig von weiteren Zeichen schwerer Störung begleitet wurde. Das Kind sah bleich und abgefallen aus und wimmerte fortwährend, die Athmung war sehr frequent und von leichtem Stöhnen begleitet, die Herzthätigkeit war verlangsamt.

Da Herr Professor Schultze sich um diese Zeit in der Anstalt befand, schritt er sofort zu der geeigneten operativen Hülfeleistung. Es wurde mit dem Katheter die Stelle aufgesucht, wo der Kanal in das Rectum umbog (siehe Fig. 1, S. 497, X), daselbst eine Falte mit der Pincette aufgehoben und mit einem Scheerenschlage eine longitudinale Spindel aus der unteren Wand des Kanales herausgeschnitten. Sofort entleerte sich ein anhaltender Strom von Meconium, welches sich in der Ampulle angesammelt zu haben schien. Eine Ausspülung der letzteren machte sich nothwendig, um die geschaffene Wunde zu übersehen.

Der Kanal war von hellrother Schleimhaut ausgekleidet, welche ohne deutliche Grenze in die Schleimhaut des Rectum überging (siehe Fig. $2 c$, S. 497). Der Scheerenschnitt hatte gerade diejenige Stelle des Kanales getroffen, wo er in das Rectum über- 
ging. Der äussere Wundrand war gleichmässig von äusserer Haut gebildet, der innere jedoch zur Hälfte von der Schleimhaut des Kanales, zur Hälfte von der Schleimhaut des Rectum.

Fig. 1.

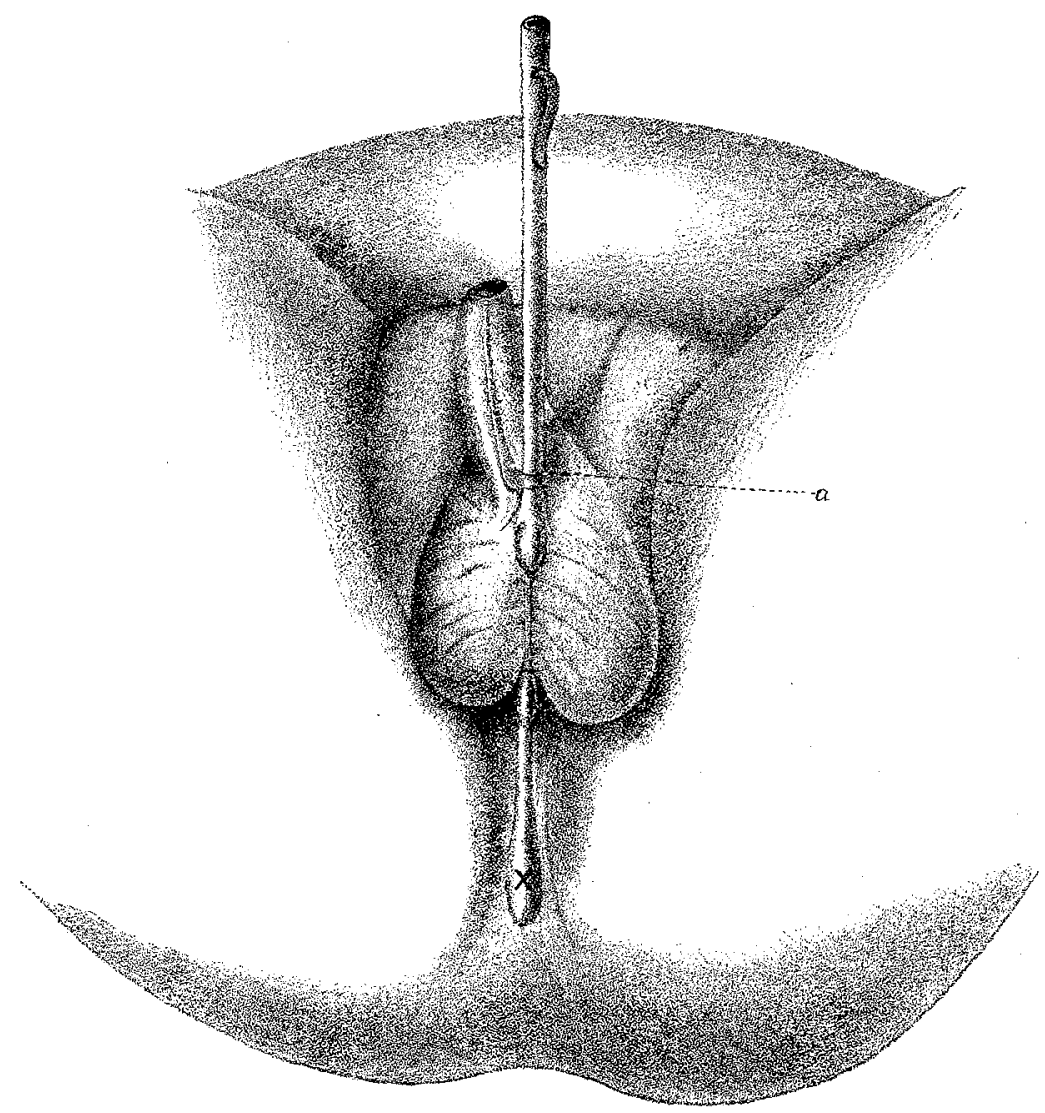

a Mündung des Kanales. $\times$ Stelle des Afters.

Hig. 2. Durch zahlreiche feine Seidenknopfnähte wurde nun, so weit es anging (von $a$ bis $b$ ), hinten die äussere Haut mit der Schleimhaut des Rectum vernäht und vorn der Rest mit derjenigen des Kanales. Der Plan war, die Wundränder so genau wie möglich zu vereinigen, damit eine Eingangspforte für Schädlichkeiten vermieden werde. Von einem Verbande wurde abgesehen.

Nach fünf Tagen (15́. April 1886) war die Wunde reactions- 
los geheilt, die Knopfnähte waren von selbst abgefallen. Der Kanal bestand noch in voller Ausdebnung und konnte mit einem Flüssigkeitsstrahle ausgespült werden.

Das Ergebniss der Operation war schon damals insoweit bemerkenswerth, als eine Füllung des Kanales mit Fäcalmassen bei der Defäcation nicht mehr beobachtet wurde, wie sie in dem von Esmarch berichteten ähnlich operirten Falle erfolgte. Ein endgültiges Urtheil über die Function konnte ich jedoch erst gewinnen, als ich 1888 den danals $2 \frac{1}{2}$ jährigen Knaben in Jena wieder untersuchte.

Der Kleine ist vollkommen continent und im Stande, die Fäces wie jedes andere Kind willkürlich zurïckzuhalten. Der Kanal besteht noch und ist ein wenig weiter, dafür aber kürzer geworden. Während er früher an der Unterseite des Penis mündete, befindet sich jetzt die Mündung an der Grenze zwischen Penis und Scrotum. Die Schleimhaut im Kanale hat noch dieselbe blassrothe Farbe wie früher, scheint indessen gar nichts abzusondern.

Dies ist die Geschichte jenes Falles.

An den soeben mitgetheilten Fall möchte ich einige Betrachtungen anknüpfen: 1) Ueber die Frage, wie die Entstehung der Verbildung entwicklungsgeschichtlich zu erklären sei; 2) über die Einreihung derselben in das System analoger Verbildungen; 3) über die Seltenheit derselben und endlich 4) über die in solchen Fällen einzuschlagende Therapie.

Man hat bisher einmüthig für die Entstehung der in Rede stehenden Missbildungen die Erklärung angenommen, welche Perls seinerzeit in folgender Weise gegeben hat: Bis zur fünften Woche des embryonalen Lebens besteht beim Menschen die Allantoiscloake. In den Stiel der Allantois, da, wo sich dieselbe aus dem Enddarme ausgestülpt hat, mündet nicht allein der Darm ein, sondern auch die Urnierengänge, die Ausführungsgänge der Harnund Geschlechtsdrüsen. Zunächst ist der einzige Ausgang dieser Cloake der Stiel der Allantois, der Urachus, und bei Persistenz dieses Zustandes kommt es entweder zur Bildung der Cloake ohne Blasenspalte, falls für die fötalen Excrete genügender Raum vorhanden ist, oder zur Bildung der Cloake mit Blasenspalte, falls die letztere platzt. ${ }^{1}$ )

Zwei Fälle, welche die Richtigkeit dieses Satzes recht treffend

1) Ahlfeld, Die Missbildungen des Menschen. Leipzig 1880. S. 232. 
darlegen, seien hier beilä日fig erwähnt. Es sind die von Winckel im Lehrbuche der Geburtshülfe S. 435, Fig. 89 abgebildeten und S. 436 beschriebenen Zwillinge. Sie befanden sich in einem gemeinsamen Amnion; der eine hatte einen Nabelschnurbruch mit Ektopia viscerum, der andere eine Spina bifida. Beide zeigten Klumpfüsse und Atresie der äusseren Genitalien. Beide besassen

Fig. 3.

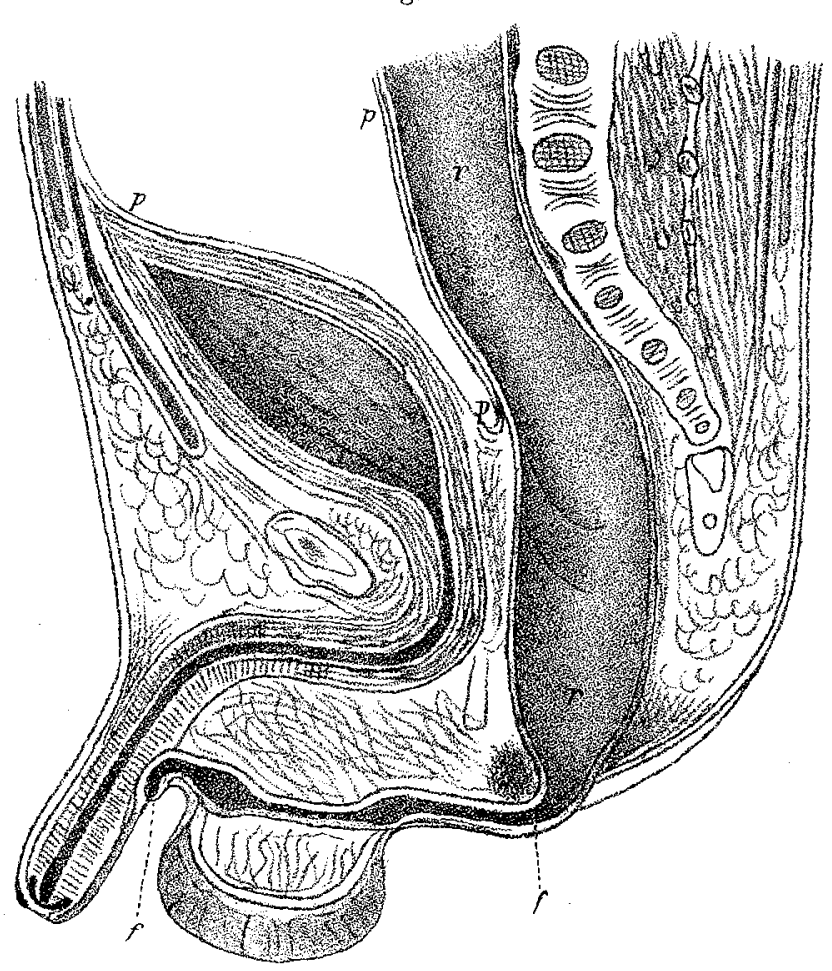

$1 / 2$ natürliche Grösse.

Sagittalschnitt, in das Schema von Esmarch eingezeichnet. $p$ Bauchfell. $r$ Rectum. $f$ Fistelgang.

Testikel, Prostata und Samenbläschen, bei beiden bestand die Allantoiscloake, bei dem einen mit Meconium gefüllt und mit dem Urachus weit in Verbindung stehend, so dass man es durch den Nabel herausdrücken konnte, bei dem anderen geplatzt und leer. Ihre Entwickelung entsprach ungefähr der 20. Woche; die Testikel fanden sich adhärent in der Nierengegend. Derartige Fälle berichtet Ahlfeld nur sechs. Sie scheinen mithin selten zu sein. 
Bei regelmässiger Entwickelung besteht dieser Zustand nicht lange. Allmälig verengt sich der Urachus, während gleichzeitig vor dem Schwanzende eine Einstülpung der äusseren Leibeswand sich bildet und dem weiter nach hinten sich ausdehnenden Enddarme entgegenwächst. Diese Grube muindet in letzteren ein, zu einer Zeit, wo er noch mit dem Stiele der Allantois in offener Verbindung steht, bildet also von nun an eine Zeit lang die Cloakenmündung, die gemeinsame Ausmündung des Darmes und Harn-Geschlechtsapparates. Allmälig rückt nun aber das Septum Douglasii, d. i. jene Falte zwischen Darm und Allantoisstiel, der späteren Blase, tiefer und tiefer und trennt endlich den Darm vom HarnGeschlechtsapparate.

Dadurch, dass sich im vierten Monate der Damm als Scheidewand noch zwischen beide schiebe, werde diese Trennung noch vollständiger.

Erleiden nun diese Vorgänge Störungen, vollzieht sich wohl die Trennung vom Harn-Geschlechtsapparate und das Tieferwachsen des Enddarmes, aber nicht das Einstülpen der äusseren Leibeswand, so kommt es zur Atresia ani. Bildet sich diese Einstülpung, aber wächst der Enddarm nicht genügend herab, oder verfehlen sich beide, so kommt es zur Atresia recti. Es kann dabei die Aftergrube bei Mädchen statt in den Mastdarm in die Scheide einmünden. Oft auch scheint After und Mastdarm secundär wieder obliterirt zu sein, dann kommt es zur Atresia ani et recti und an Stelle von beiden findet sich eine feste Zellgewebsmasse oder ein dünner muskulöser Strang.

Tritt dagegen das Septum Douglasii nicht oder nur unvollständig tiefer, so kommt es zu widernatürlichen Ausmündungen des Mastdarmes in die Nachbarorgane.

Tritt es gar nicht tiefer, bleibt die ursprüngliche Verbindung bestehen, so besteht ein Anus vesicalis; tritt sie etwas tiefer, so bildet sich bei Knaben ein Anus urethralis, bei Mädchen, wo sich die Derivate der Müller'schen Gänge dazwischen schieben, ein Anus uterinus ${ }^{1}$ ) oder vaginalis.

Während gegen die Richtigkeit der entwicklungsgeschichtlichen Erklärung der bisher betrachteten Missbildungen wenig einzuwenden ist, so erscheint eine Gruppe von Verbildungen, welche

1) Ein Fall ist von Esmarch beobachtet, erwähnt in „Die Krankheiten des Mastdarmes und des Afters". Deutsche Chirurgie von Billroth-Lücke, 1887. S. 25. 
sich unmittelbar anschliesst und von den Forschern bisher auf die gleiche Weise erklärt wurde, nach neueren Untersuchungen über die Entstehung des Dammes in einem anderen Lichte.

Um die bisherige Anschaung (Perls) zu charakterisiren, lasse ich Ahlfeld ${ }^{1}$ ) selbst sprechen, welcher die allgemein geltende Erklärung in folgenden Worten wiedergiebt: „Bei noch tieferem Herabdrängen der abnormen Afteröffnung fällt die Mündung in die Raphe des Scrotum beim Knaben, Anus scrotalis, in die äussere Fläche der Vulva beim Mädchen, Anus vulvalis. Endlich in den extremsten Fällen zeigt sich die Oeffnung im Perineum, Anus perinealis."

Dieser Erklärungsweise liegt offenbar die Anschauung zu Grunde, dass auch der Damm, welcher sich ja zwischen Darm und Harn-Geschlechtssystem hineinschieben sollte, sich an das Șeptum Douglasii anschliesse und von oben herabwachse.

Wenn nun Paul Reichel ${ }^{2}$ ), gestützt auf seine Untersuchungen über die Entwickelung des Dammes, die Theorie von Perls schon für diejenigen Fälle von Anus vaginalis, wo die Einmündung im untersten Theile der Vagina sich findet, nicht gelten lassen kann, sondern annimmt, dass in diesen Fällen, wo ja auch die Mündung oft recht weit ist, die abnorme Einmündung einfach dadurch bestehen bleibt, dass die Dammanlage sich mit dem Septum Douglasii nicht vereinigte, so muss mit noch grösserem Rechte eine andere Erklärungsweise gesucht werden für eine Gruppe von Missbildungen des Anus, welche mit dem Septum Douglasii gar nichts zu thun haben kann, da bei ihnen die Trennung zwischen Darm und Harn-Geschlechtsapparat eine vollständige und nur die Ausmündung mehr oder weniger weit nach vorn an den Damm oder die äusseren Genitalien verlegt ist.

Es umfasst diese Gruppe den Anus perinealis, den Anus vulvalis, den Anus scrotalis und den Anus suburethralis. Die letzte Bezeichnung gebrauche ich meines Wissens zum ersten Male. Die betreffenden Fälle wurden bisher mit dem Anus urethralis zusammengeworfen und ebenso benannt, trotzdem der abnorm mündende Anus durchaus nichts weiter mit der Urethra gemein hat, als dass ein Fistelgang mehr oder weniger weit unter der Urethra am Penis entlang verläuft, bevor er ausmündet. Mit mehr Recht

1) Die Missbildungen des Menschen. Leipzig 1880. S. 235, Zeile 24.

2) Zeitschrift für Geburtshülfe und Gynäkologie, Bd. XIV, S. 82. Archiv f. Gynäkologie. Bd. XXXIV. Hft. 3. 
könnte man noch die Bezeichnung Anus penilis oder ähnlich gebrauchen und nur aus Rücksichten auf den Wohllaut wählte ich obige Bezeichnung.

Bevor ich die vorliegende Missbildung entwicklungsgeschichtlich zu erklären versuche, ist es nothwendig, dass ich in Kürze die Ergebnisse der Untersuchungen Paul Reichel's über die Entwickelung des Dammes kurz darlege. In den Lehrbüchern der Entwickelungsgeschichte findet man entweder die Angabe, dass die Entwickelung des Dammes noch nicht genügend untersucht sei, oder die Autoren drücken sich ausweichend und unsicher aus: der Damm schiebe sich um die zehnte Woche zwischen Darm und Harn-Geschlechtsapparat oder Aehnliches. Nur in wenigen findet man die Angabe, dass der Damm aus zwei seitlich von der Wand der Cloakenmündung entspringenden und in der Mitte der wachsenden Falten entstehe. So beschreibt O. Hertwig, Lehrbuch der Entwickelungsgeschichte, Jena 1886, S. 298 den Vorgang wie folgt: ,Es beginnt nämlich die Scheidewand (das Septum Douglasii), durch welche in der Tiefe der Sinus urogenitalis und die Oeffnung des Mastdarmes gegen einander abgegrenzt sind, nach aussen vorzuwuchern, wobei gleichzeitig a uch an der Seitenwand der Cloake Falten entstehen und sich mit ihr vereinigen." Leider findet sich keine Angabe der Quelle dieser Darstellung im Literaturverzeichnisse. Paul Reichel hat nun gefunden, dass diese Darstellung vollkommen richtig und schon im Jahre 1830 von Rathke ausgesprochen worden ist; nur bilden sich jene Falten erst dadurch, dass zwei Höcker (Analhöcker) hinter der Cloakenmündung sich bilden, seitlich auseinander und nach vorn wachsen, mit dem. hinteren Ende der Genitalfalten sich vereinigen. Mit diesen vereint wachsen sie sich von beiden Seiten entgegen, um nach oben mit dem Septum Douglasii in Verbindung zu treten.

Ich sehe ab von den mehr theoretischen Erwägungen Reichel's, welche sich hauptsächlich gegen die Anschauung wenden, dass der Damm lediglich durch Herabwachsen des Septum Douglasii aus diesem letzteren entstehe. Er erblickt gegen diese einen Beweis darin, dass der Damm die Merkmale des äusseren Keimblattes besitze, das Septum Douglasii jedoch stamme rom inneren Keimblatte ab. Daraus und aus dem Umstande, dass am Damme eine Raphe vorhanden sei, am Hinterdamme und über dem Kreuzbeine keine, gehe vielmehr hervor, dass der Damm durch Ver- 
schmelzung zweier seitlicher Anlagen aus dem Ektoderm entstehe. Die hiermit bekämpfte Anschauung ist streng genommen von keinem der Forscher vertreten worden und nur indirect in der Erklärung eines Theiles der Missbildungen des Afters nach Perls enthalten.

Indem ich mich in der Darstellung der von Reichel beschriebenen Entwickelungsart an die von ihm gegebenen Figuren halte, glaube ich, dass nur wenig hinzuzufügen sein wird, um mich verständlich zu machen. In Fig. 1 giebt Reichel den Befund von einem menschlichen Embryo aus dem Beginne der siebenten Woche. Die Cloakenmündung ist rautenförmig, die Genitalfalten noch nicht entwickelt, der Genitalhöcker noch nicht in Glans und Schaft getrennt, die Genitalfurche wenig ausgeprägt. Der Genitalhöcker bildet mit seiner Basis die vordere Begrenzung der Grube, in dessen Tiefe man das Septum Douglasii gewahren kann. Die hintere Begrenzung wird von zwei Höckern gebildet, von Reichel zuerst beschrieben und Analhöcker genannt. Sie sind lange nicht so hoch als das Schwanzende des Körpers, das spätere Kreuz- und Steissbein, welches jene überragt; Fig. $1 a$ stellt dieselben Verhältnisse im Sagittalschnitte dar. Fig. 2 stellt das nächsthöhere Stadium dar; das Alter der Frucht ist leider nicht angegeben. Der Genitalhöcker zeigt Schaft und Eichel und eine tiefere Genitalfurche. An seiner Basis befinden sich die Genitalfalten. Die Analhöcker sind mit dem Schwanzende fast gleich hoch und nach seitwärts hin stärker entwickelt. Sie treten eben in Beziehung zum hinteren Ende der Genitalfalten. In Fig. 3 ist das nächstfolgende Stadium gezeichnet. Die beiden Stellen, wo Analhöcker und Genitalfalten sich berührt haben und verschmolzen sind, sind von beiden Seiten medianwärts zusammengetreten und haben gemeinsam den Damm gebildet. Nach oben zu sind sie gleichzeitig mit dem Septum Douglasii in Beziehung getreten. Der After ist so gleichsam von den Analhöckern umrahmt worden und seine vorher quere Oeffnung ist in einen Längsspalt verwandelt worden. Dies wäre ungefähr die Form, aus welcher sich die bleibende Gestalt der weiblichen Genitalien unschwer ableiten lässt; die weitere Entwickelung zum männlichen Typus durch Verwachsen der Genitalfalten ist bekannt.

Mit Hülfe dieser entwicklungsgeschichtlichen Darstellung lässt sich nun die in Rede stehende Gruppe von Missbildungen, zu welcher der mitgetheilte Fall gehört, sehr leicht erklären. Die 
Analhöcker standen vielleicht von vornherein sehr. nahe bei einander und standen auch dann, als sie nach seitwärts und vorwärts auseinander wuchsen, noch in so inniger Beziehung, dass sie eine beide bedeckende Hautbrücke mit nach vorn und den Genitalfalten entgegen trugen. Durch Verwachsen der so gebildeten beiden Dammanlagen wurde nun die Oeffnung, welche schon durch die ursprüngliche Einstülpung, die schon um die vierte Woche sich gebildet hatte und die gemeinsame Cloakenmündung gewesen war, in ihrem hinteren Abschnitte secundär wieder verschlossen. Das andrängende Meconium hat nun das untere Ende des Darmes weiter und weiter nach vorn ausgestülpt, und je weiter nun die Genitalfalten schon verwachsen waren, desto weiter nach vorn wurde die Mündung verlegt. Nicht der höchsie Grad, wie bei der Theorie von Perls, sondern der geringste ist nach dieser Auffassung der Anus perinealis, der zweite der Anus vulvalis beim weiblichen, der Anus scrotalis beim männlichen Geschlechte; der dritte und höchste Grad ist dann der Anus suburethralis, wolcher begreiflicherweise nur beim männlichen Geschlechte vorkommen kann. Nur so lässt es sich erklären, dass der Sphinkter ani vorhanden ist und dass der Darm durch ihn ausmündet, wie das in den hierher gehörigen Fällen gefunden worden ist. So wurde in dem Falle von $K_{0} \mathrm{ch}^{1}$ ) durch die Obduction festgestellt, dass der Sphinkter den Darm umschloss, während in dem Falle von Giese $^{2}$ ) der Afterschliessmuskel sein Vorhandensein durch seine Contractionen anzeigte. Im vorliegenden Falle wurde letzteres ebenfalls beobachtet, ausserdem beweist das functionelle Ergebniss die Richtigkeit dieser Annahme. Der Knabe ist vollkommen continent.

Eine vorwärts treibende $\mathrm{Kraft}$, vielleicht peristaltische Bewegung, müsste ziemlich früh im fötalen Darme thätig sein, um die fistelartige Ausdehnung des Darmendes nach vorn zu bewirken. Uebrigens fehlt es in der Literatur nicht an Beispielen, welche dies zu bestätigen scheinen. So berichtet Thomas Bartholin ${ }^{3}$ ) von einem kräftigen 40jährigen Manne, welcher ohne After und Ruthe mit gespaltener Harnblase den Koth von Zeit zu Zeit durch ein in den Mund eingeführtes Horn entleerte. Auch Baux sah (Meckel) ein 14jähriges Mädchen, welches, ohne After, Harn-

1) Monatsschrift für Geburtskunde, Bd. XXXI, S. 18.

2) Nene Zeitschrift für Geburtskunde, Bd. I, S. 131. Bei Reichel, a. a. 0 ., S. 85 .

3) Histoire anatomique, Cent. V, observ. 65, p. 113. 
und Geschlechtsöffnung geboren, den Koth durch den Mund entleerte, den Harn aber durch die Brüste entleert haben soll. Aufklärung über diese Fälle giebt, wie $\mathrm{Esmarch}^{1}$ ) meint, ein von Bils anatomisch untersuchter Fall, wo bei einem Fötus der Darm aus dem Unterleibe sich wieder aufwärts wendete, durch die Brusthöhle (vor dem Oesophagus) und die Mundhöhle verlaufend neben der Mundöffnung ausmündete.

Das Zusammentreffen der Missbildung des Afters mit der Ueberzahl der Endphalanx des rechten Daumens bestätigt die alte Erfahrung, dass sich häufig mehrere Missbildungen gleichzeitig finden. Gewiss ist hier nicht an eine dem Individuum innewohnende Neigung zu Ueberflussbildungen zu denken.

Bei einem oberflächlichen Einblicke in die specielle Literatur 'fand ich auch, dass schon $\mathrm{Crame} \mathrm{r}^{2}$ ) einen Fall von Atresia recti mit Hyperdaktylie gepart mitgetheilt hat. Dort fanden sich an jeder Hand sechs Finger, an jedem Fusse sechs Zehen und eine einfache Atresia ani gleichzeitig vor.

Soweit über die Entwickelungsgeschichte dieses Falles. Die Stelle im System der angeborenen Missbildungen des Afters, in welches er eingereiht werden muss, ergiebt sich nun von selbst.

I. Einfacher Verschluss des Enddarmes

a) durch Mangel der normalen Hauteinstülpung, Atresia ani;

b) durch ungenügendes Tiefertreten des Enddarmes bei vorhandener Analportion, Atresia recti;

c) durch secundäre Obliteration, Atresia recti et ani.

II. Verschluss des Enddarmes mit abnormer Ausmündung (Anus praeternaturalis congenitus)

a) durch mangelhaftes Tiefertreten des Septum Douglasii Einmündung in den Harn-Geschlechtsapparat, Anus vesicalis, urethralis, uterinus, vaginalis;

b) durch Störungen bei Entwickelung des Dammes und der äusseren Genitalien und abnorme Mündung im Verlaufe derselben, Anus vulvo-vaginalis, perinealis, scrotalis, vulvalis, suburethralis.

Hinsichts der Benennung der Missbildung der zweiten Haupt-

1) a. a. O., S. 28.

2) Casper's Wochenschrift für die gesammte Heilkunde 1834, Nr. 51. 
gruppe habe ich mich Ahlfeld") angeschlossen. Papendorf hatte die Bezeichnung Atresia ani vesicalis, vaginalis u. s. w. eingeführt. Ahlfeld fand diese Bezeichnungsweise für $z u$ lang und unzutreffend und wendete die zweckmässige Bezeichnungsweise der Chirurgen beim Anus praeternaturalis auf diese Fälle an. Entsprechend dem Anus lumbalis und inguinalis unterscheidet er einen Anus vesicalis, vaginalis u. s. w. Schon fünf Jahre früher hatte $\mathrm{K}$ üster ${ }^{2}$ ) dieselbe Bezeichnungsweise gebraucht, aber wiederum ein überflüssiges Wort beigefügt. Er wollte die Bezeichnung Anus fistulosus vesicalis u. s. w. angewendet wissen.

Ueber die Häufigkeit oder richtiger Seltenheit der vorliegenden Verbildung ist es sehr schwer, ein richtiges Urtheil zu gewinnen, will man nicht die Herkulesarbeit auf sich nehmen, jeden einzelnen Fall von Atresia ani in der Literatur daraufhin nachzulesen. Wie oben gesagt, sind die Fälle von Anus suburethralis in den Sammelwerken ausnahmelos mit dem Anus urethralis zusammengeworfen, oder aber sie finden sich, wie der obenerwähnte Fall von Giese, welcher mit dem meinigen vollständig übereinstimmt, unter der Bezeichnung Anus scrotalis. Immerhin gewinnt man ein Bild von der Seltenheit dieser Fälle, wenn ein Geburtshelfer wie Winckel, der weit über 20000 Geburten beobachtet hat, nur einen einzigen derartigen Fall, einen Anus hymenalis, erlebte, während nach Collins (Esmarch) auf der Entbindungsanstalt zu Dublin unter 16654 Geburten nur einmal überhaupt eine Aftermissbildung beobachtet wurde. Dabei hat Curling ${ }^{3}$ ) 100 Fälle von angeborenen Missbildungen des Afters und Mastdarmes des operativen Ausganges wegen zusammengestellt, darunter fand sich Atresia ani $26 \mathrm{Mal}$, Atresia recti in 31 Fällen, $26 \mathrm{Mal}$ bestand Anus vesicalis oder urethralis, $11 \mathrm{Mal}$ Anus vaginalis und nur in 6 Fällen mündete der After äusserlich an abnormer Stelle, und zwar betraf es vier Knaben und zwei Mädchen. Von letzteren zeigte eine Anus vulvalis und eine Anus perinealis, von den Knaben waren zwei mit Anus perinealis, zwei mit Anus scrotalis behaftet.

Einen Fall von Anus suburethralis höchsten Grades hat Cru-

1) a. a. 0., S. 235.

2) Berliner klinische Wochenschrift 1875 , S. 456 .

3) Inquiry into Treatment of congenital imperfections of the Rectum etc. Medico-chirargical Transactions 1860, p. 271. 
veilhier ${ }^{1}$ ) beschrieben, einen zweiten berichtet Witt $^{2}$ ); bei beiden mündete der Anus an der Glans penis unter der Urethra.

In Bezug auf die Therapie kann ich mich kurz fassen, da das Wesentliche schon oben in der Geschichte des Falles mitgetheilt ist. Der Erfolg zeigt, dass es nicht immer nöthig ist, den ganzen Kanal zu spalten, wie Esmarch a. a. O, S. 40 empfiehlt. Nicht in allen Fällen hat ein schonenderes Verfahren die üblen Folgen wie in dem Falle von Beck, den Esmarch mittheilt. Bei einem so operirten 17 jährigen Manne bestand ein Divertikel, welches bis in das Scrotum führte und deu jungen Mann dadurch belästigte, dass es sich bei jeder Defäcation mit Koth füllte. Hätte man in unserem Falle den ganzen Kanal gespalten, so wäre die Wunde am Scrotum, wo der Kanal $11 / 2 \mathrm{~cm}$ tief von der Oberfläche verlief, doch recht breit und vor Sepsis schwer zu schützen gewesen. Zuerst galt es die Lebensgefahr zu beseitigen; wären noch Unbequemlichkeiten zurïckgeblieben, so hätte man sie vielleicht jetzt beseitigen können, wo das Individuum soweit erwachsen ist, dass ein aseptischer Verband einigermassen anzubringen wäre und auch geduldet würde. Da der Kanal durchaus keine Beschwerden macht, ja die Eltern gar nicht wussten, dass er noch besteht, werden sie sich schwerlich noch zu einer Operation entschliessen.

Herrn Professor Schultze sage ich für das Material meinen besten Dank.

1) Anatomie pathologique, Livr. I, B. 6, Fig. 6 und 7. Förster, Missbildungen, Taf. XXIII, Fig. 7 und 8.

2) De atresia ani congenita anatomica. Inangural-Dissertation. Kiel 1859. Operirt von Middeldorpff in Breslau. 